\title{
Optimized control for dynamical performance of the polishing robot in unstructured environment
}

\author{
Zhong Luo*, Shuxian Yang, Yilan Sun and Hongyi Liu \\ School of Mechanical Engineering and Automation, Northeastern University, Shenyang, 110004, China
}

Received 12 February 2010

Revised 21 June 2010

\begin{abstract}
In this paper, the dynamical performance of polishing robot working in unstructured environment is investigated. Structure mechanism and operating principle of a polishing robot are introduced firstly, and its dynamical model is established. Then, a S-shaped acceleration-deceleration path planning method and a human-simulated intelligent control (HSIC) strategy are proposed. The S-shaped acceleration-deceleration path planning method is to switch the magnitude and direction of the abrupt velocities between motion sections in order to improve the work efficiency, the smoothness of movement and the processing accuracy. The HSIC control strategy is built based on the unstructured environment information measured by ultrasonic sensors, in which the appropriate programs prepared in advance are determined according to the size and sign of both the control error and its change rate. Simulation results show that the intelligent control strategy combining with optimum path planning method are effective to reduce the structure vibration, to improve the stability and the control accuracy of the polishing robot system.
\end{abstract}

Keywords: Polishing robot, unstructured environment, path planning, human-simulated intelligent control (HSIC)

\section{Introduction}

A polishing robot is an important automatic machine for the purpose of finishing process to obtain required metal surface quality and shape accuracy of a workpiece, and it is widely used in space, aviation, manufacturing industry and so on [1-3]. The working environment of the polishing robot is a typical unstructured environment, such as the workpiece surface is unknown in advance and can not be described in mathematical expression. For the reason of the unstructured environment, it is often difficult to ensure the finishing accuracy in work, and spends much time and needs well-experienced operators mostly. So it is meaningful to develop novel kinds of polishing robotic equipment [4-6]. For the existing machines for surface finishing process, many of them are the conventional machine tools [7,8], the classical robots [9-11] and the parallel robots [12,13], and so on. The industrial robot based polishing system has many advantages such as big workspace and good flexibility, but its finishing plan and track of free-form surface are difficult. The CNC machine tool has good trajectory error but not acceptable error when polishing irregular surfaces. The parallel polishing machine is usually faster and more rigid than a traditional polishing robot but its workspace is usually more limited [14].

At present, much works about the polishing process research used for polishing with bound abrasives has been done, such as the polishing expert system integrated with sensor information [6,15], an intelligent polishing system using an acoustic emission-based intelligent monitoring scheme, and the optimization of process parameters based

${ }^{*}$ Corresponding author: Zhong Luo, School of Mechanical Engineering and Automation, Northeastern University, Shenyang, 110004, China. Tel.: +86 24 83680540; E-mail: zhluo@mail.neu.edu.cn. 
Table 1

Main technical parameters of the system

\begin{tabular}{lc}
\hline Parameters & Values \\
\hline Range of shell's internal diameter $(\mathrm{mm})$ & $300 \sim 600$ \\
Range of shell's length $(\mathrm{mm})$ & $600 \sim 3000$ \\
Speed of big arm's movement $(\mathrm{mm} / \mathrm{s})$ & 3 \\
Speed of polishing head(r/s) & 50 \\
Linear speed of rotating bracket $(\mathrm{mm} / \mathrm{s})$ & $30 \sim 32$ \\
Polishing accuracy $(\mathrm{mm})$ & $<0.04$ \\
Temperature $\left({ }^{\circ} \mathrm{C}\right)$ & $-20 \sim 70$ \\
\hline
\end{tabular}

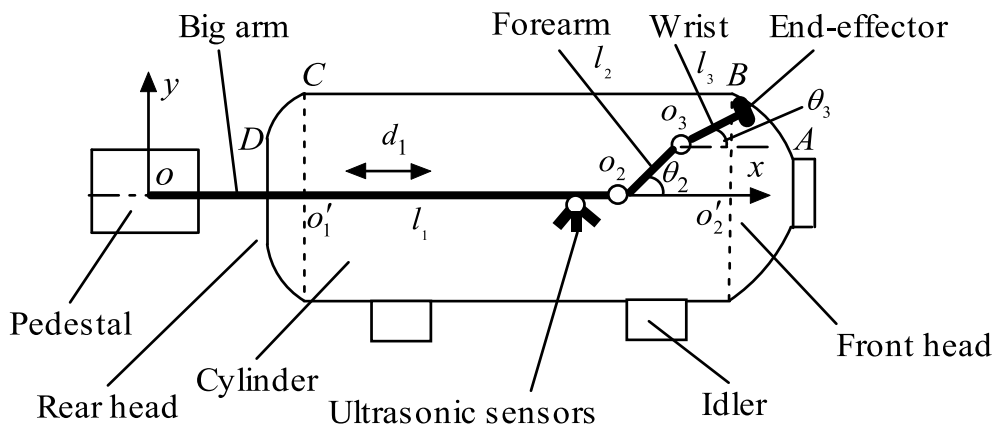

Fig. 1. Structure diagram of polishing robot system.

on the Taguchi method [11]. A robotic die polishing workstation with controller PC can exchange the polishing tool automatically so that the operation of it is completely unmanned [16]. The contact stress for an automated polishing process was modeled and analyzed, which determined the quality of the polished part, not the force exerted on the polishing tool [17]. A high precision polishing robot with a learning-based hybrid position/force controller was developed for polishing PET (Poly Ethylene Terephthalate) bottle molds [18]. An automatic mold polishing system was presented in $[19,20]$, which integrates with the mold geometry process kernel, path planner, process planner, and the force control robot. For a robotic polishing system for mold manufacturing reported in [21], the automatic planning and programming system is developed based on CAD data.

In this paper, the artificial intelligent based optimal control a polishing robot in unstructured environment is studied with especially considering the goal of the structure vibration and machining accuracy. Firstly, the main structure mechanism of the polishing robot is presented, and the dynamical model of it is established. The designing of the controller with practical components is presented. Then, a S-shaped acceleration-deceleration path planning method is propsed. A HSIC strategy is built based on the unstructured environment information measured by ultrasonic sensors, which is to summarize human control experience, imitate human control behavior, and describe the control strategies by the mathematical rules. Finally, the conclusions are made.

\section{Practical setup and kinetic equations of the polishing robot}

As shown in Fig. 1, a polishing robot is divided into two parts: the main body and the deputy body. The deputy body is a rotating bracket that drives the workpiece of a thin shell structure to rotate around the $x$-axis. The position of the end-effector in the plane of xoy is determined by the big arm (in translating motion) and the forearm (in rotating motion). The wrist (in rotating motion) ensures the end-effector contract with work surface in tangent direction.

The working principle of the robot is depicted in Fig. 2. The practical setup is shown in Fig. 3. Main technical parameters of the system are listed in Table 1.

There are three ultrasonic rangefinders on the big arm, numbered as 1,2 and 3, and their length is $b$. The sensor 1 is fixed on the big arm in the direction $y$. The sensors 2 and 3 are for exploring the inclined planes. The last two sensors can swing driven by a servo motor, where $o$ ' and $\psi$ denote the swing centre and the angle between the axes of the two sensors respectively. 


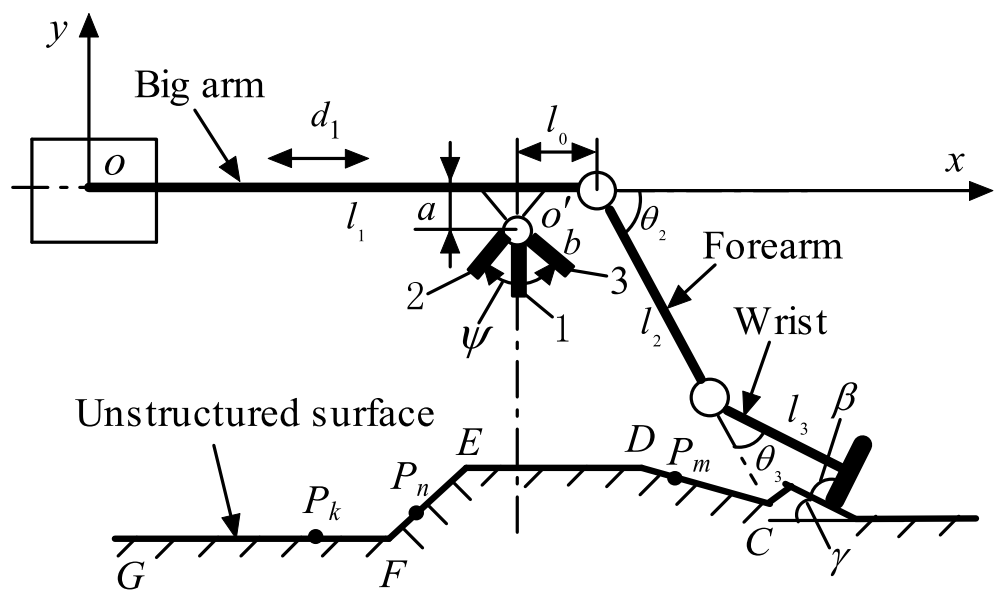

Fig. 2. Structure model of main body in unstructured environment.

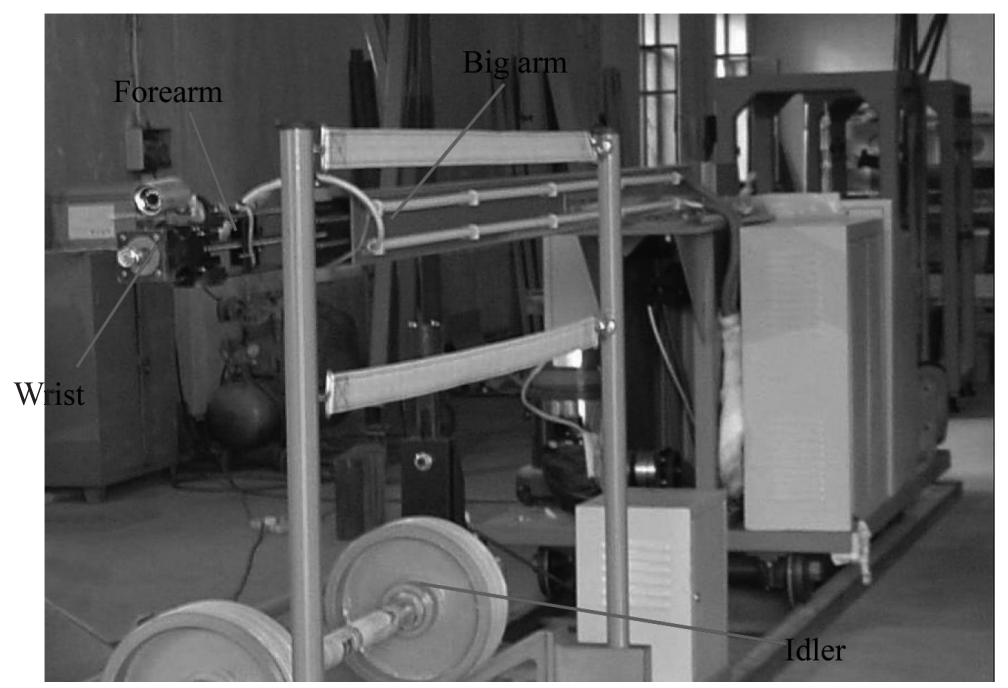

Fig. 3. Experimental setup.

The three degrees of freedom of the robot are: the displacement of the big arm $d_{1}$, and the rotational joints of $\theta_{2}$ and $\theta_{3}$. Besides, $\beta$ represents the angle between the axis of the end-effector and the tangent of the environmental surface, and $\gamma$ denotes the angle between the tangent of the environmental surface and the $x$-axis, and $a$ represents the distance between the point $o^{\prime}$ and the big arm, and $l_{0}$ denotes the distance between the point $o^{\prime}$ and the joint of the big arm and the forearm. The lengths of the big arm, forearm and the wrist are $l_{1}, l_{2}$ and $l_{3}$, respectively.

The equations of each joint variable of the robot by kinematics inverse transformation are as follows

$$
\left\{\begin{array}{l}
d_{1}(t)=x(t)-l_{2}\left(1-\cos \theta_{2}(t)\right)-l_{3}(1-\cos \psi(t))+r \sin \psi(t) \\
\theta_{2}(t)=\arcsin \left(\frac{1}{l_{2}}\left(y(t)-l_{3} \sin \psi(t)-r \cos \psi(t)\right)\right) \\
\theta_{3}(t)=\theta_{2}(t)-\psi(t)
\end{array}\right.
$$

where $\psi(t)=\gamma(t)+\pi / 2-\beta(t)$.

The structure diagram of the robot is shown in Fig. 4. The Masses of the big arm, forearm and wrist are respectively $m_{1}, m_{2}$ and $m_{3}$, and their corresponding position vectors are respectively $\mathbf{r}_{1}, \mathbf{r}_{2}$ and $\mathbf{r}_{3}$.

The position vectors are

$$
\mathbf{r}_{1}=\left(d_{1}+l_{1}\right) \mathbf{i}
$$




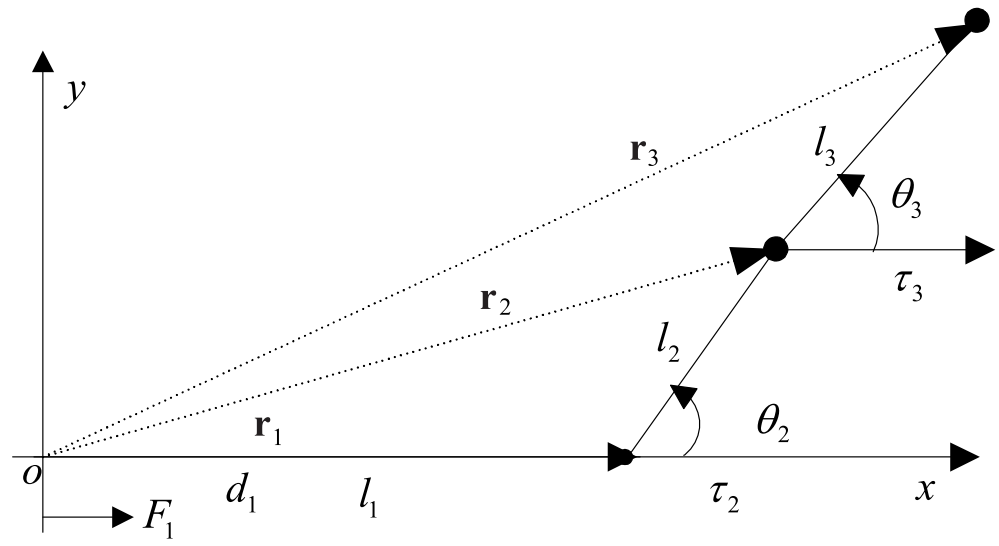

Fig. 4. The structure diagram of main body.

$$
\begin{aligned}
\mathbf{r}_{2} & =\mathbf{r}_{1}+\left(l_{2} \cos \theta_{2}\right) \mathbf{i}+\left(l_{2} \sin \theta_{2}\right) \mathbf{j} \\
& =\left(l_{1}+d_{1}+l_{2} \cos \theta_{2}\right) \mathbf{i}+\left(l_{2} \sin \theta_{2}\right) \mathbf{j} \\
\mathbf{r}_{3} & =\mathbf{r}_{2}+\left(l_{3} \cos \theta_{3}\right) \mathbf{i}+\left(l_{3} \sin \theta_{3}\right) \mathbf{j} \\
& =\left(l_{1}+d_{1}+l_{2} \cos \theta_{2}+l_{3} \cos \theta_{3}\right) \mathbf{i}+\left(l_{2} \sin \theta_{2}+l_{3} \sin \theta_{3}\right) \mathbf{j}
\end{aligned}
$$

Then, the velocity vectors $\mathbf{v}_{1}, \mathbf{v}_{2}$ and $\mathbf{v}_{3}$ are as follows

$$
\begin{aligned}
& \mathbf{v}_{1}=\frac{\mathrm{d} \mathbf{r}_{1}}{\mathrm{~d} t}=\left(\dot{x}_{1}\right) \mathbf{i} \\
& \mathbf{v}_{2}=\left(\dot{d}_{1}-l_{2} \dot{\theta}_{2} \sin \theta_{2}\right) \mathbf{i}+\left(l_{2} \dot{\theta}_{2} \cos \theta_{2}\right) \mathbf{j} \\
& \mathbf{v}_{3}=\left(\dot{d}_{1}-l_{2} \dot{\theta}_{2} \sin \theta_{2}-l_{3} \dot{\theta}_{3} \sin \theta_{3}\right) \mathbf{i}+\left(l_{2} \dot{\theta}_{2} \cos \theta_{2}+l_{3} \dot{\theta}_{3} \cos \theta_{3}\right) \mathbf{j}
\end{aligned}
$$

The kinetic energy equation of system is

$$
\begin{aligned}
\mathbf{K}= & \frac{1}{2} m_{1} \mathbf{v}_{1}^{2}+\frac{1}{2} m_{2} \mathbf{v}_{2}^{2}+\frac{1}{2} m_{3} \mathbf{v}_{3}^{2} \\
= & \frac{1}{2}\left(m_{1}+m_{2}+m_{3}\right) \dot{d}_{1}^{2}+\frac{1}{2}\left(m_{2}+m_{3}\right) l_{2}^{2} \dot{\theta}_{2}^{2}+\frac{1}{2} m_{3} l_{3}^{2} \dot{\theta}_{3}^{2} \\
& -\left(m_{2}+m_{3}\right) l_{3} \dot{d}_{1} \dot{\theta}_{2} \sin \theta_{2}-m_{3} l_{3} \dot{d}_{1} \dot{\theta}_{3} \sin \theta_{3}+m_{3} l_{2} l_{3} \dot{\theta}_{2} \dot{\theta}_{3} \cos \left(\theta_{3}-\theta_{2}\right)
\end{aligned}
$$

The potential energy referencing to the coordinate origin is

$$
\begin{aligned}
\mathbf{P} & =m_{1} g \mathbf{r}_{1}+m_{2} g \mathbf{r}_{2}+m_{3} g \mathbf{r}_{3}=m_{2} g l_{2} \sin \theta_{2}+m_{3} g\left(l_{2} \sin \theta_{2}+l_{3} \sin \theta_{3}\right) \\
& =\left(m_{2}+m_{3}\right) g l_{2} \sin \theta_{2}+m_{3} g l_{3} \sin \theta_{3}
\end{aligned}
$$

The dissipative energy of the system is

$$
\mathbf{D}=\frac{1}{2} c_{1} \dot{d}_{1}^{2}+\frac{1}{2} c_{2} \dot{\theta}_{2}^{2}+\frac{1}{2} c_{3}\left(\dot{\theta}_{3}-\dot{\theta}_{2}\right)^{2}
$$

The total work done by the external force is

$$
\mathbf{W}=F_{1} d_{1}+\tau_{2} \theta_{2}+\tau_{3} \theta_{3}
$$

With the Lagrange principle of the following 


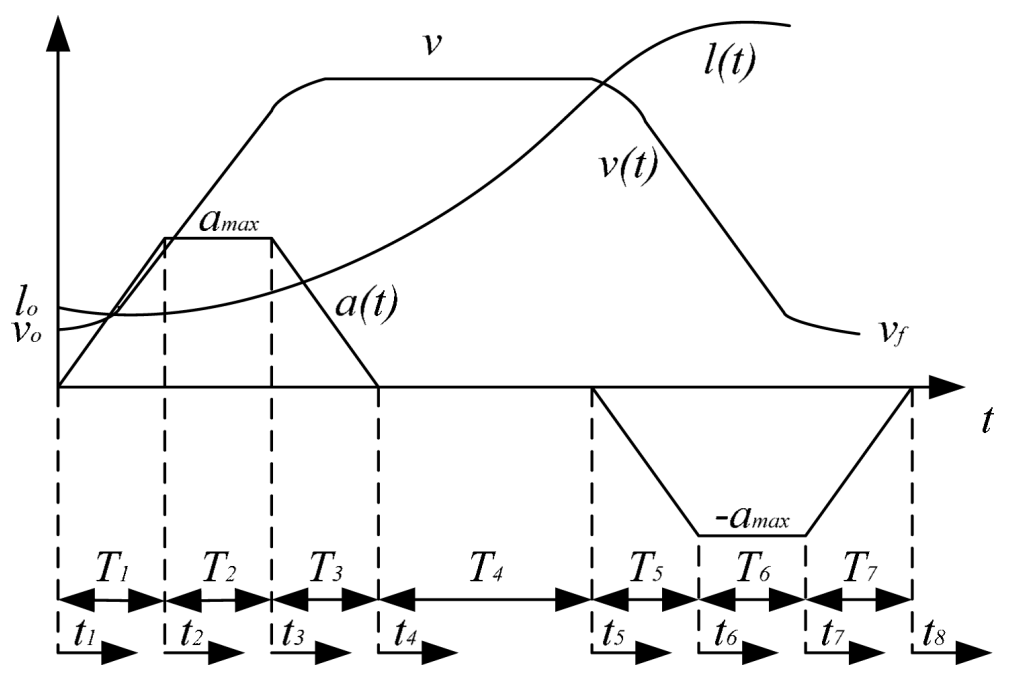

Fig. 5. Planning drawing of method of S-shaped acceleration and deceleration.

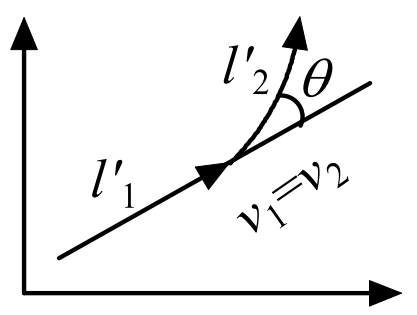

Fig. 6. Switching mode of velocity.

$$
\frac{\partial \mathbf{W}}{\partial q_{i}}=\frac{\mathrm{d}}{\mathrm{d} t} \frac{\partial \mathbf{K}}{\partial \dot{q}_{i}}-\frac{\partial \mathbf{K}}{\partial q_{i}}+\frac{\partial \mathbf{D}}{\partial \dot{q}_{i}}+\frac{\partial \mathbf{P}}{\partial q_{i}}, i=1,2, \cdots, n
$$

We can obtain the system's kinetic equation as follows

$$
\left\{\begin{aligned}
F_{1}= & \left(m_{1}+m_{2}+m_{3}\right) \ddot{d}_{1}-\left(m_{2}+m_{3}\right) l_{2}\left(\ddot{\theta}_{2} \sin \theta_{2}+\dot{\theta}_{2}^{2} \cos \theta_{2}\right)-m_{3} l_{3}\left(\ddot{\theta}_{3} \sin \theta_{3}+\dot{\theta}_{3}^{2} \cos \theta_{3}\right)+c_{1} \dot{d}_{1} \\
\tau_{2}= & \left(m_{2}+m_{3}\right)\left(l_{2} \ddot{\theta}_{2}-\ddot{d}_{1} \sin \theta_{2}\right) l_{2}+m_{3} l_{2} l_{3} \ddot{\theta}_{3} \cos \left(\theta_{3}-\theta_{2}\right)-m_{3} l_{2} l_{3} \dot{\theta}_{3}\left(\dot{\theta}_{3}-2 \dot{\theta}_{2}\right) \sin \left(\theta_{3}-\theta_{2}\right) \\
& +\left(c_{2}+c_{3}\right) \dot{\theta}_{2}-c_{3} \dot{\theta}_{3}+\left(m_{2}+m_{3}\right) g l_{2} \cos \theta_{2} \\
\tau_{3}= & -m_{3} l_{3}\left[\ddot{d}_{1} \sin \theta_{3}-l_{2} \ddot{\theta}_{2}-l_{2} \dot{\theta}_{2}^{2} \sin \left(\theta_{3}-\theta_{2}\right)\right] m_{3} l_{3}^{2} \ddot{\theta}_{3}+c_{3}\left(\theta_{3}-\theta_{2}\right)+m_{3} g l_{3} \cos \theta_{3}
\end{aligned}\right.
$$

\section{Planning method for S-shaped acceleration-deceleration path}

The planning for the acceleration-deceleration path of the polishing robot carried out in Cartesian coordinate space to ensure the motion trajectory of the end-effector keeping in strict accordance with the required goal. Shown in Fig. 5, $a_{\max },-a_{\max }, v, v_{o}, v_{f}$ and $l_{o}$ are the maximum acceleration, maximum deceleration, average feed velocity, initial velocity, end velocity and initial displacement.

In the S-shaped acceleration-deceleration planning, one of the motion segments is divided into seven time intervals of $\left(T_{1} \sim T_{7}\right)$. The corresponding both feed velocity and acceleration are planned as continuous functions with time variable. With this planning method, the vibration and shock of the robot can be suppressed effectively [3].

Considering the motion trajectory of the end-effector $A B+B C+C D$ shown in Fig. 1, there are two ellipsoid ends tangent to the junctions on cylinder and with the same direction of velocity. The switching modes of velocity are all straight line segments, where the direction in arc segments is invariant as shown in Fig. 6. The end-effector doesn't 
Table 2

The program results of S-shaped acceleration-deceleration planning

\begin{tabular}{lcccccccc}
\hline Regional number & \multicolumn{2}{c}{ The first section } & & \multicolumn{2}{c}{ The second section } & & \multicolumn{2}{c}{ The third section } \\
\cline { 2 - 3 } & $T(\mathrm{~ms})$ & $l(\mu \mathrm{m})$ & & $T(\mathrm{~ms})$ & $l(\mu \mathrm{m})$ & & $T(\mathrm{~ms})$ & $l(\mu \mathrm{m})$ \\
\hline 1 & 50 & 417 & & 25 & 1302 & & 0 & 0 \\
2 & 0 & 0 & & 0 & 0 & & 0 & 0 \\
3 & 50 & 2083 & & 25 & 1797 & & 0 & 0 \\
4 & 7530 & 376500 & & 11260 & 843800 & & 7500 & 376500 \\
5 & 0 & 0 & & 25 & 1797 & & 30 & 1787 \\
6 & 0 & 0 & & 0 & 0 & & 20 & 563 \\
7 & 0 & 0 & & 25 & 1302 & & 30 & 220 \\
\hline
\end{tabular}

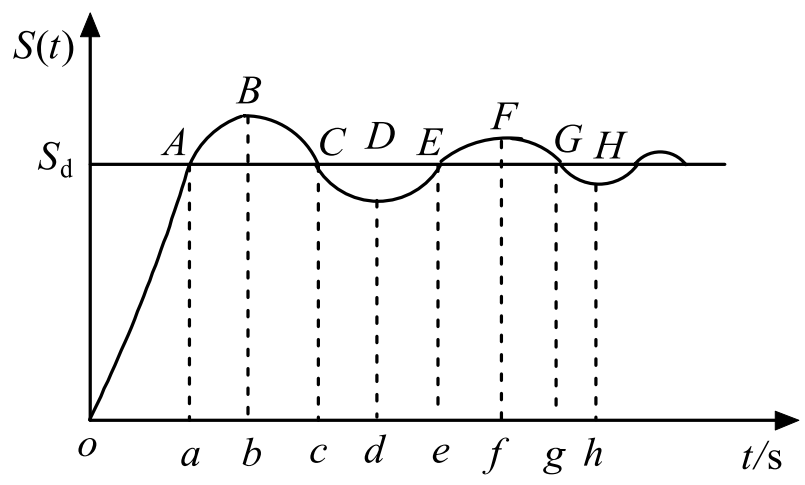

Fig. 7. Step response curve of typical dyadic system.

need to decelerate to zero and then accelerate at three trajectory junctions. In this way, the whole polishing process is fast, smooth, and continuous. In Fig. $6, l_{1}^{\prime}, l_{2}^{\prime}$ are respectively the lengths of current linear motion section and next arc one; $v_{1}, v_{2}$ are respectively the closure velocity of current motion section and the initial velocity of next one.

The precision and smoothness of the motion trajectory of the end-effector along the shell's internal wall is critical for achieving accurate polishing. Table 2 shows the program result of the S-shaped acceleration-deceleration of the polishing robot, where the longest transiting time is that of the accelerating in the first motion section, where $T_{a c}=T_{1}+T_{2}+T_{3}=100 \mathrm{~ms}$. Meanwhile, the maximum polishing length is $l\left(T_{d e}\right)=l\left(T_{5}+T_{6}+T_{7}\right)=$ $3.1 \mathrm{~mm}$, which is the section of accelerating and decelerating during the second motion section. Compared with the whole polishing process, the above time and length of transiting section are extremely short. The new proposed planning method not only ensures the polishing efficiency but also provides the precondition guarantees for the next transformation accuracy of the trajectory coordinates during programming.

\section{Human-simulated intelligent control strategy}

The control strategy of the polishing robot hopes that the end-effectors' motion trajectory and working profiles are completely consistent and the contact force keeps constant. But the tracking error exists certainly, especially in the unstructured enviroment. The method of human-simulated intelligent control (HSIC) is presented in this paper. In HSIC, the control variables are adjusted in real-time according to the position error and its change rate between the desired control force and the contact force of the end-effector referring to the workpiece.

If the robot system of trajectory tracking and position control can be simplified as a linear system, the HSIC can be described by using a step response function shown in Fig. 7.

The characteristic variables of HSIC are important for the dynamic process of the robot, and their signs (negative or positive) are listed in Table 3.

In order to describe the dynamic characteristics of HSIC, two errors are defined as follows.

The error between the desired position and the actual position is 
Table 3

Symbol change of characteristic variables

\begin{tabular}{lcccccccc}
\hline & $\mathrm{OA}$ & $\mathrm{AB}$ & $\mathrm{BC}$ & $\mathrm{CD}$ & $\mathrm{DE}$ & $\mathrm{EF}$ & $\mathrm{FG}$ & $\mathrm{GH}$ \\
\hline$e_{n}$ & $>0$ & $<0$ & $<0$ & $>0$ & $<0$ & $<0$ & $<0$ & $>0$ \\
$\Delta e_{n}$ & $<0$ & $<0$ & $>0$ & $>0$ & $>0$ & $<0$ & $>0$ & $>0$ \\
$e_{n} \cdot \Delta e_{n}$ & $<0$ & $>0$ & $<0$ & $>0$ & $<0$ & $>0$ & $<0$ & $>0$ \\
\hline
\end{tabular}

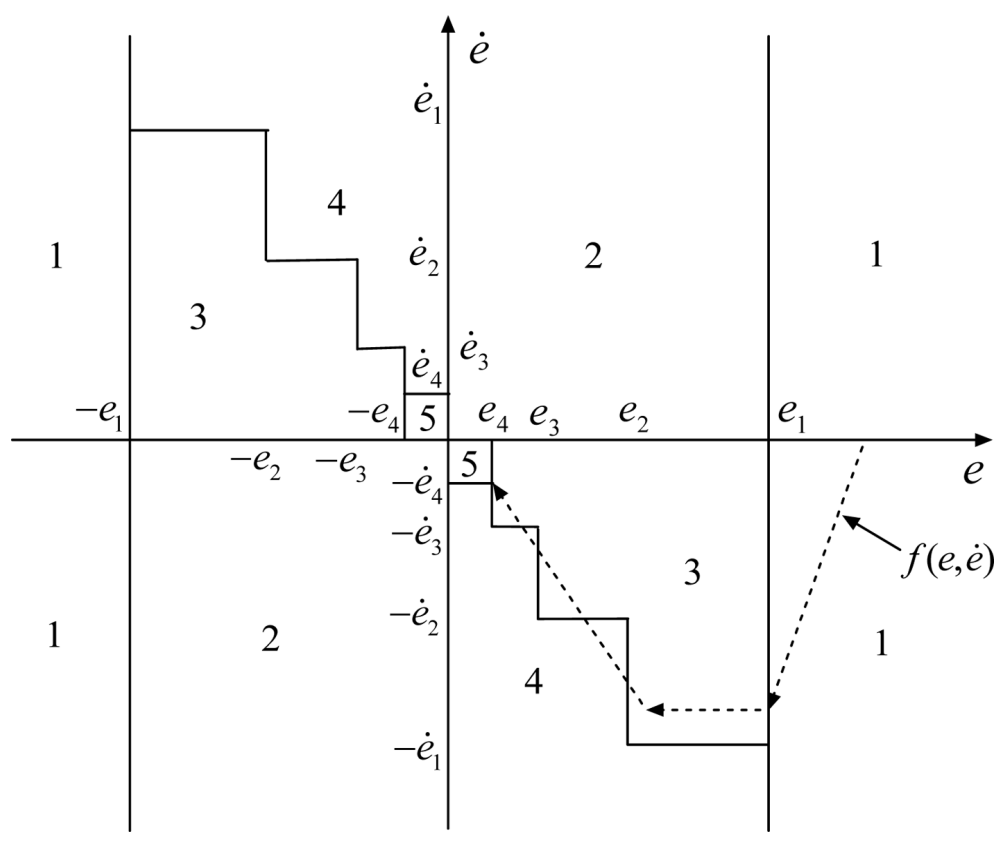

Fig. 8. Ideal trajectory of force error of HSIC.

$$
e=S_{d}-S_{e}
$$

where $S_{d}$ is the desired position of robot system and $S_{e}$ is the actual position.

The change rate of the position error is

$$
\Delta e_{n}=e_{n}-e_{n-1}, \Delta e_{n-1}=e_{n-1}-e_{n-2}
$$

where $e_{n}$ is the position error at the $n$th sampling period; $e_{n-1}$ is the position error at the $(n-1)$ th sampling period; and $e_{n-2}$ is the position error at the $(n-2)$ th sampling period.

The position changing trend is described and forecasted based on the negative or positive signs of the above characteristic variables. If $e_{n} \cdot \Delta e_{n}<0$, systematic development changes towards trend of error diminution, such as $O A, B C, D E$ and $F G$ in Fig. 7, which indicates there are the extreme values. Contrarily, if $e_{n} \cdot \Delta e_{n}>0$, the process changes towards trend of error aggrandizement, such as $A B, C D, E F$ and $G H$, which indicates there is no extreme value. The characteristic variable $\Delta\left(\Delta e_{n}\right)$ represents the change rate of the position error. As is shown in Fig. 7, it is an overshoot part for $A B C$ part because of $\Delta\left(\Delta e_{n}\right)>0$, and an undershoot part for $C D E$ part because of $\Delta\left(\Delta e_{n}\right)$ $<0$. In the process of establishing trajectory tracking model, the signs of $e_{n}, e_{n} \cdot \Delta e_{n-1}$ and $\Delta e_{n}$ are identified and used to recognize the dynamic behaviors of the trajectory in real time.

In order to improve the effectiveness of the trajectory tracking with a HSIC algorithm, the ideal trajectory of the position error is given and shown in Fig. 8.

The dotted lines in Fig. 8 indicate the desired target trajectory $f(e, \dot{e})$. The thresholds' values of position error are $e_{1}, e_{2}, e_{3}$ and $e_{4}$, and their change rates are $\dot{e}_{1}, \dot{e}_{2}, \dot{e}_{3}$ and $\dot{e}_{4}$, correspondingly. The above eight parameters are determined according to the actual requirements of the robot. Different control development processes in the five regions in Fig. 8 are as follows:

1) While the position error is bigger, the corresponding region 1 adopts as far as possible big control action. 
2) While the position error increases, the corresponding region 2 uses PID (Proportion - Integral - Differential) control.

3) While the position error decreases and tends to target slowly, the corresponding region 3 applies proportional control.

4) While the position error decreases and tends to target rapidly, the corresponding region 4 adopts PD (proportion - differential) control.

5) When the position error and the change rate of the error all satisfy the request, the corresponding region 5 keeps the current control model.

According to above regulation, the characteristic elements of trajectory tracking of the HSIC control can be expressed as

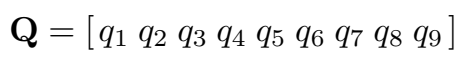

where, $q_{1}: e_{n} \cdot \Delta e_{n} \leqslant 0 ; q_{2}:\left|e_{n}\right| \geqslant e_{1} ; q_{3}:\left|e_{n}\right| \geqslant e_{2} ; q_{4}:\left|e_{n}\right| \geqslant e_{3} ; q_{5}:\left|e_{n}\right| \geqslant e_{4} ; q_{6}:\left|\dot{e}_{n}\right| \geqslant \dot{e}_{1} ; q_{7}:\left|\dot{e}_{n}\right| \geqslant \dot{e}_{2}$; $q_{8}:\left|\dot{e}_{n}\right| \geqslant \dot{e}_{3} ; q_{9}:\left|\dot{e}_{n}\right| \geqslant \dot{e}_{4}$.

The characteristic model is expressed as

$$
\Phi=\left[\phi_{1} \phi_{2} \phi_{3} \phi_{4} \phi_{5}\right]
$$

where, $\phi_{1} \Rightarrow\left[q_{2}\right] ; \phi_{2} \Rightarrow\left[\bar{q}_{1} \cap \bar{q}_{2}\right]$;

$$
\begin{aligned}
\phi_{3} & \Rightarrow\left[\left(q_{1} \cap \bar{q}_{2} \cap q_{5} \cap \bar{q}_{8}\right) \cup\left(q_{1} \cap \bar{q}_{2} \cap q_{4} \cap \bar{q}_{7} \cap q_{8}\right) \cup\left(q_{1} \cap \bar{q}_{2} \cap q_{3} \cap \bar{q}_{6} \cap q_{7}\right)\right] ; \\
\phi_{4} & \Rightarrow\left[\left(q_{1} \cap \bar{q}_{2} \cap q_{3} \cap q_{6}\right) \cup\left(q_{1} \cap \bar{q}_{3} \cap q_{7}\right) \cup\left(q_{1} \cap \bar{q}_{4} \cap \bar{q}_{7} \cap q_{8}\right) \cup\left(q_{1} \cap \bar{q}_{5} \cap \bar{q}_{8} \cap q_{9}\right)\right] ; \\
\phi_{5} & \Rightarrow\left[q_{1} \cap \bar{q}_{5} \cap \bar{q}_{9}\right] .
\end{aligned}
$$

The control model is expressed as

$$
\Psi=\left[\psi_{1} \psi_{2} \psi_{3} \psi_{4} \psi_{5}\right]
$$

where, $\psi_{1} \Rightarrow u_{n}=K_{p} \cdot e_{n}+K_{d} \cdot \dot{e}_{n}+K_{i} \int e_{n} \mathrm{~d} t ; \psi_{2} \Rightarrow u_{n}=\operatorname{sgn}\left(e_{n}\right) \cdot U_{\max }$;

$\psi_{3} \Rightarrow u_{n}=K_{p} \cdot e_{n}+K_{d} \cdot \dot{e}_{n} ; \quad \psi_{4} \Rightarrow u_{n}=K_{p} \cdot e_{n} ; \quad \psi_{5} \Rightarrow u_{n}=u_{n-1}$.

where, $u_{n}$ is the controller output at the $n$th sampling period; $u_{n-1}$ is the controller output at the $(n-1)$ th sampling period; $K_{p}$ is the proportion coefficient; $K_{d}$ is the differential coefficient; $K_{i}$ is the integral coefficient; $U_{\max }$ is the maximum controller output value.

The inference rule is expressed as

$$
\Omega=\left[\omega_{1} \omega_{2} \omega_{3} \omega_{4} \omega_{5}\right]
$$

where, $\omega_{1}: \phi_{1} \Rightarrow \psi_{2} ; \omega_{2}: \phi_{2} \Rightarrow \psi_{1} ; \omega_{3}: \phi_{3} \Rightarrow \psi_{4} ; \omega_{4}: \phi_{4} \Rightarrow \psi_{3} ; \omega_{5}: \phi_{5} \Rightarrow \psi_{5}$.

\section{Example}

Take a third-order system as an example to study the HSIC control strategy presented in this paper, where the system is the same as that in [22]. Its transfer function is

$$
G(s)=\frac{523500}{s^{3}+87.35 s^{2}+10470 s}
$$

Supposing the sampling time is $1 \mathrm{~ms}$ and using Z-transform, Eq. (20) can be re-written as

$$
\begin{aligned}
\operatorname{yout}(k)= & -\operatorname{den}(2) \text { yout }(k-1)-\operatorname{den}(3) \text { yout }(k-2)-\operatorname{den}(4) \text { yout }(k-3) \\
& +\operatorname{num}(2) u(k-1)+\operatorname{num}(3) u(k-2)+\operatorname{num}(4) u(k-3)
\end{aligned}
$$




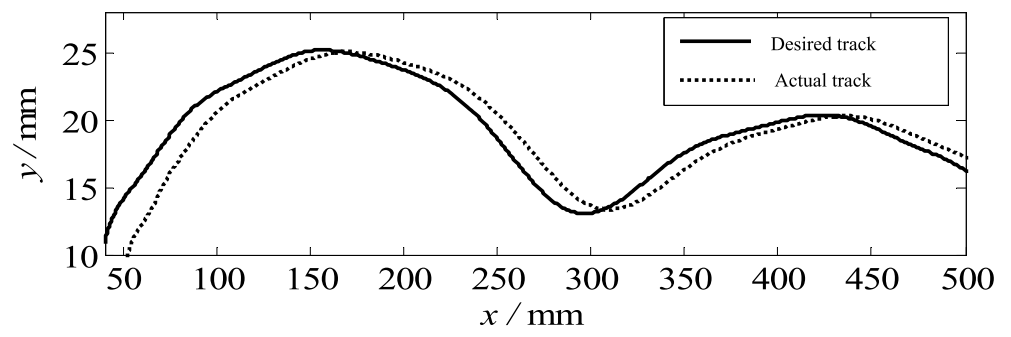

Fig. 9. Simulated tracking curve of PID control.

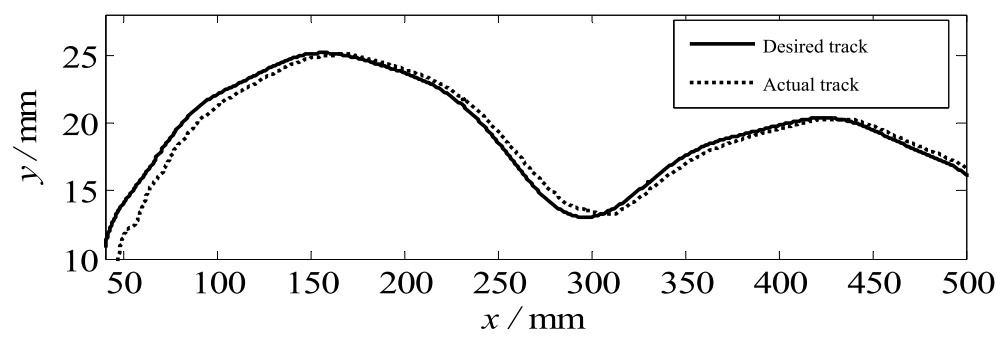

Fig. 10. Simulated tracking curve of HSIC.

If with PID control, the simulation results are shown in Fig. 9, where the parameters are $K_{p}=0.5, K_{i}=0.001$, $K_{d}=0.001$. The average track error between the desired track and the actual track is $\Delta y_{a v e}=3.26 \mathrm{~mm}$.

If with the HSIC control, where the PID parameters are used the same, the maximum controller output is $U_{\max }=$ $10 \mathrm{~mm}$, and the thresholds of force error and their change rate are assumed as:

$$
\begin{aligned}
& e_{1}=15 \mathrm{~mm}, \quad e_{2}=10 \mathrm{~mm}, \quad e_{3}=5 \mathrm{~mm}, \quad e_{4}=1 \mathrm{~mm} \\
& \dot{e}_{1}=10 \mathrm{~mm}, \quad \dot{e}_{2}=5 \mathrm{~mm}, \quad \dot{e}_{3}=1 \mathrm{~mm}, \quad \dot{e}_{4}=0.5 \mathrm{~mm}
\end{aligned}
$$

The simulated result is shown in Fig. 10, where the average track error is $\Delta y_{\text {ave }}=1.51 \mathrm{~mm}$.

Comparing Fig. 10 with Fig. 9, it is apparent that the effect of HSIC tracking method is better than PID control method in the same settings of control parameters. Therefore, the HSIC strategy is adopted in the polishing robot under unknown environment, as long as the parameters of HSIC are determined based on the actual requirements. In our practice, HSIC method is effective in working efficiency and accuracy of the contact force, and restrains the error enhancing ultimately.

\section{Conclusions}

The S-shaped acceleration-deceleration path planning method and human-simulated intelligent control (HSIC) strategy are investigated in the paper to improve the dynamic performance of the polishing robot. The S-shaped acceleration-deceleration path planning method is to switch magnitude and direction of the connection velocity among motion sections. The HSIC control strategy is well defined and the numerical simulations indicate that it is effective for a given example where the average tracking error is much smaller than that of PID control.

\section{Acknowledgements}

The work is financially supported by the Liaoning Science and Technology Research Project (No. 20091021) and the State Key Laboratory of Robotics Research Projects (No. RL0200903, RLO200808). 


\section{References}

[1] L.A. Nguyen, I.D. Walker and R.J. Defigueiredo, Dynamic control of flexible kinematically redundant robot manipulators, IEEE Transactions on Robotics and Automation 8(6) (1992), 759-767.

[2] S.G. Yue, Weak-vibration configurations for flexible robotic manipulators with kinematic redundancy, Mechanism and Machine Theory 35(2) (2000), 165-178.

[3] Y.L. Sun, H.Y. Liu, P. Wang and Z. Luo, Design of missile inner-wall grinding robot and its trajectory planning, China Mechanical Engineering 20(7) (2009), 838-843.

[4] Z. Luo, H.Y. Liu, Y.L. Sun et al., Study on contact force control based on detection by ultrasonic sensors, Journal of Northeastern University: Natural Science 29(3) (2008), 378-382.

[5] J.H. Ahn, M.C. Lee, H.D. Jeong et al., Intelligently automated polishing for high quality surface formation of sculptured die, Journal of Materials Processing Technology 13(1) (2002), 339-344.

[6] J.H. Ahn, Y.F. Shen, H.Y. Kim et al., Development of a sensor information integrated expert system for optimizing die polishing, Robotics and Computer Integrated Manufacturing 17 (2001), 269-276.

[7] X. Pessoles and C. Tournier, Automatic polishing process of plastic injection molds on a 5-axis milling center, Journal of Materials Processing Technology 20(9) (2009), 3665-3673.

[8] X.J. Wu, Y. Kita and K. Ikoku, New polishing technology of free form surface by GC. Journal of Materials Processing Technology 18(8) (2007), 81-84.

[9] T. Furukawa and D.C. Rye, Dissanayake M W. Automated polishing of an unknown threedimensional surface, Robotics and ComputerIntegrated Manufacturing 12(3) (1996), 261-270.

[10] N. Fusaomi, H. Tetsuo, H. Zenku et al., CAD/CAM-based position/force controller for amould polishing robot, Mechatronics 17 (2007), $207-216$.

[11] M.J. Tsai and J.F. Huang, Efficient automatic polishing process with a new compliant abrasive tool, International Journal of Advanced Manufacturing Technology 30 (2006), 817-827.

[12] C. Brecher, S. Lange, M. Merz et al., NURBS based ultra-precision freeform machining, Annals of the CIRP 55(1) (2006), 547-550.

[13] L. Liao, F.F. Xi and K.F. Liu, Modeling and control of automated polishing/deburring process using a dual-purpose compliant toolhead, International Journal of Machine Tools and Manufacture 48(12-13) (2008), 1454-1463.

[14] G.L. Wang, Y.Q. Wang, J. Zhao et al., Process optimization of the serial-parallel hybrid polishing machine tool based on artificial neural network and genetic algorithm, Journal of Intelligent Manufacturing (2010), DOI: 10.1007/s10845-009-0376-5.

[15] C.W. Lee and Y.C. Shin, Modeling of complex manufacturing processes by hierarchical fuzzy basis function networks with application to grinding processes, Journal of Dynamic Systems, Measurement, and Control, Transactions of the ASME 126 (2004), 880-890.

[16] B.S. Ryuh, S.M. Park and G.R. Pennock, An automatic tool changer and integrated software for a robotic die polishing station, Mechanism and Machine Theory 41 (2006), 415-432.

[17] A. Roswell, F.J. Xi and G. Liu, Modelling and analysis of contact stress for automated polishing, International Journal of Machine Tools and Manufacture 46 (2006), 424-435.

[18] F. Nagata, Y. Kusumoto, K. Watanabe et al., Polishing robot for PET bottle molds using a learning-based hybrid position/force controller, 5th Asian Control Conference (2004), 914-921.

[19] M.J. Tsai, J.J. Fang and J.L. Chang, Robotic path planning for an automatic mold polishing system, International Journal of Robotics and Automation 19(2) (2004), 81-89.

[20] M.J. Tsai, J.L. Chang and J.F. Haung, Development of an automatic mold polishing system, IEEE Transactions on Automation Science and Engineering 2(4) (2005), 393-397.

[21] J.J. Márquez, J.M. Pérez, J. Rios et al., Process modeling for robotic polishing, Journal of Materials Processing Technology 15(9) (2005), 69-82.

[22] J.K. Liu, Advanced PID control MATLAB simulation (the second edition). Beijing: Electronic Industry Press, (2004), 449-451. 

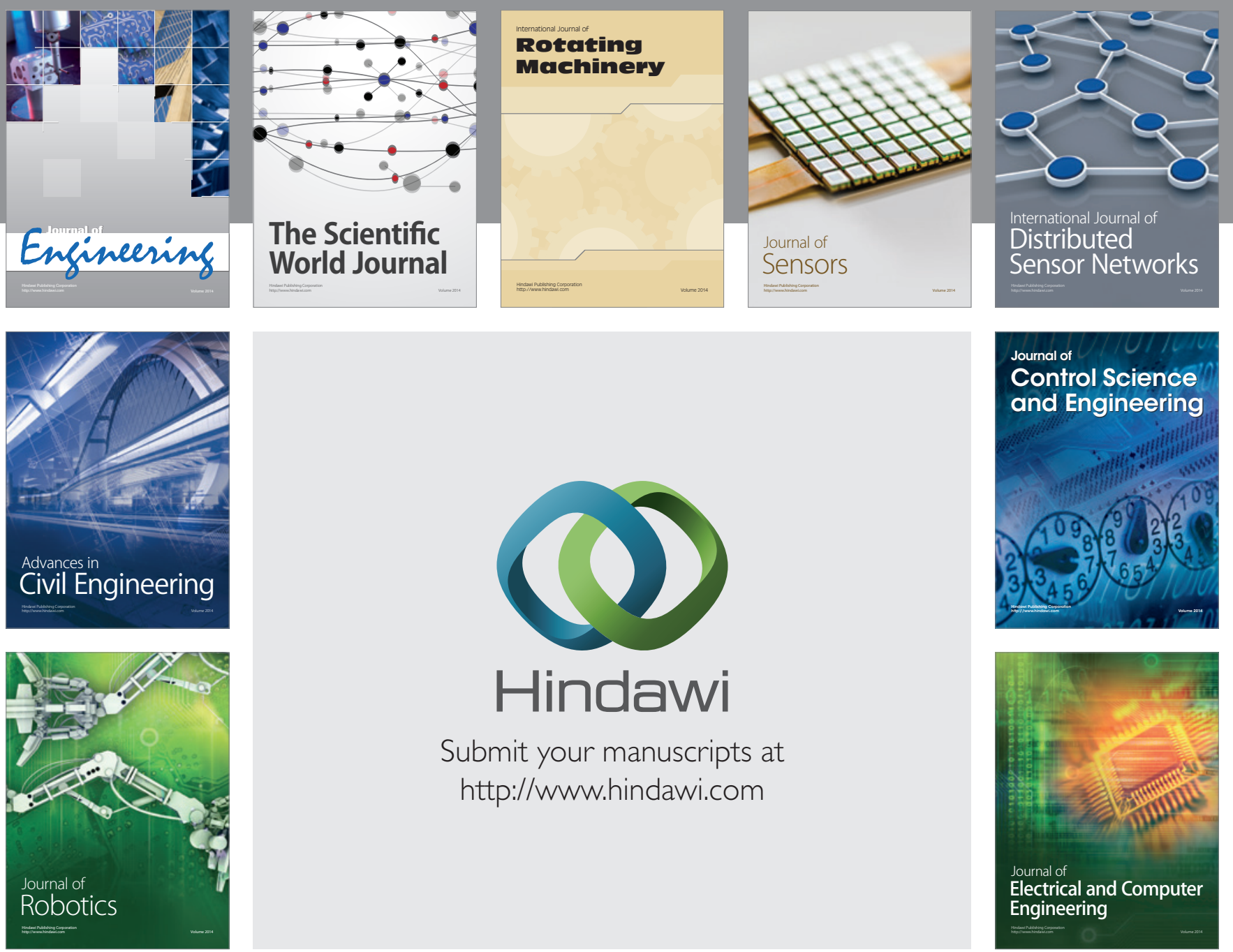

Submit your manuscripts at

http://www.hindawi.com
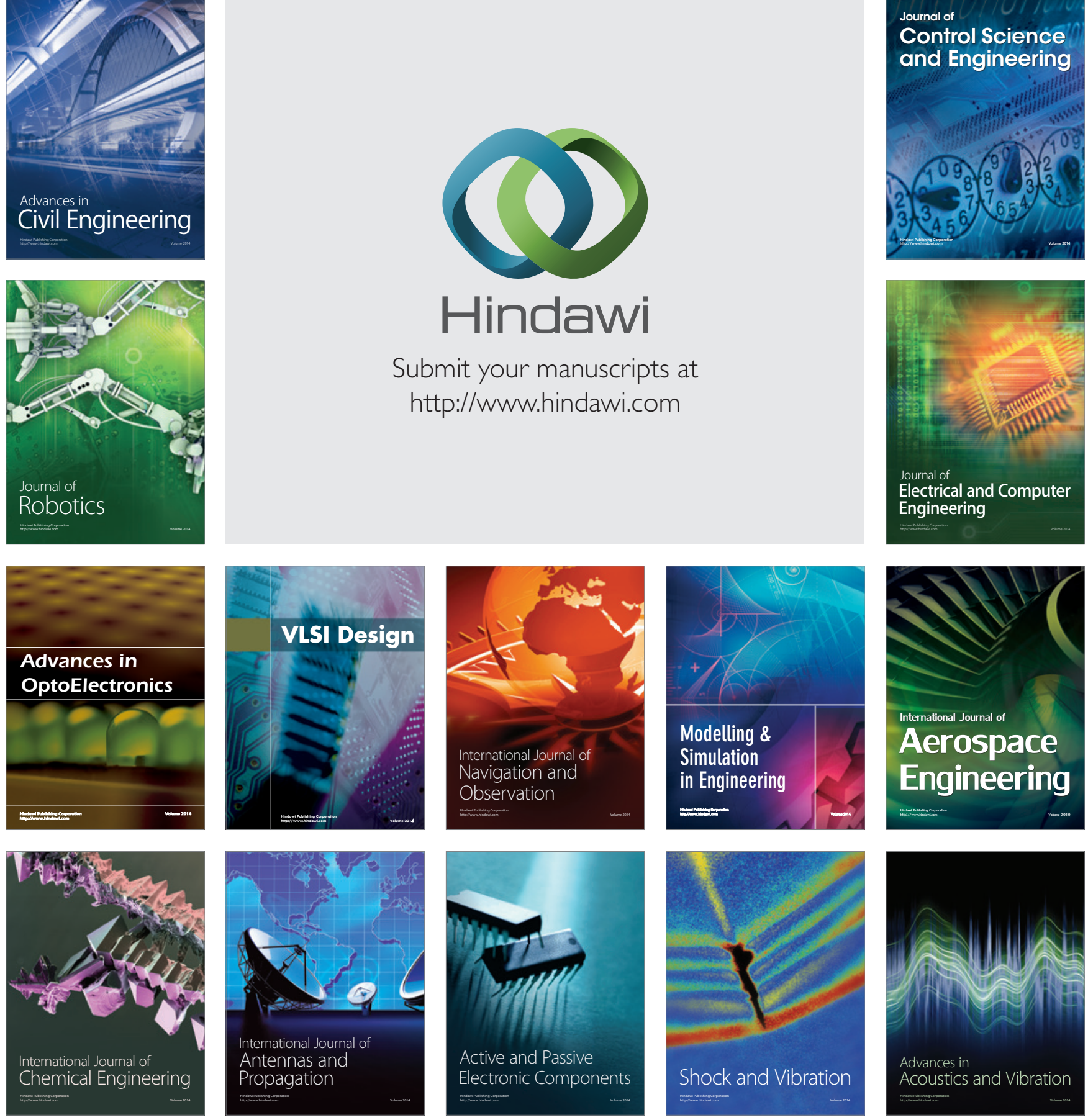